\title{
Chapter 25 \\ How We Got to QCD Matter \\ from the Hadron Side: 1984
}

\section{Rolf Hagedorn}

\begin{abstract}
Rolf Hagedorn reminisces in 1984 about limiting temperature, the development of the statistical bootstrap model (SBM). He argues that consideration of hadrons of finite size allowed the generalization of SBM into a sophisticated relativistic van der Waals-type gas, leading on to a theory of phase transformation from melting hadrons to boiling quarks.
\end{abstract}

\subsection{Introduction}

I have 50 min and 25 pages of print available to describe the origin and development of work which took 27 years and was done by perhaps more than 200 people in perhaps more than 300 papers. This leaves me about 2 min of talk per year of work and about three lines of print per paper.

You, and hopefully even those whose work is not reported here, will understand my dilemma: I have to concentrate on the essentials and skip all technical details.

I hope to be able to let you participate in retrospection in the ups and downs of this adventure, which started from a simple statistical model of hadron production without any attempt to understand the underlying dynamics and without any idea of what finally it would lead to: a phase transition to QCD matter.

This whole history can be squeezed into one sentence coined by Helmut Satz [1]: "Hadron thermodynamics defines its own limits." But how, and why? To realize and formalize that took a long time, as I shall now describe.

Invited Lecture at QM3 (Helsinki 1984); Lect. Notes Phys. 221 (1985) pp. 53-76.

Reproduced with kind permission of (C) Springer Science+Business Media, 1985.

R. Hagedorn: (deceased) CERN-TH, 1211 Geneve 23, Switzerland

J. Rafelski (ه)

Department of Physics, The University of Arizona, Tucson, AZ 85721, USA

(C) The Author(s) 2016 


\subsection{Pre-bootstrap}

In 1957, F. Cerulus and I were asked by the then CERN-TH-Division leader, B. Ferretti, to do a few calculations ("just a fortnight") of particle production at the future CERN $30 \mathrm{GeV}$ proton synchrotron. We used the Fermi statistical model [2] and realized at once that the phase space integrals could not be calculated with the known approximations ( $m \rightarrow 0$ and $m \rightarrow \infty$ ) since, on the average, the particles (mostly pions) would be neither relativistic nor non-relativistic. We heard of the Monte Carlo method and adapted it to our problem [3]. We thus were able to compute reliably hundreds of phase space integrals with all the then known hadrons and resonances. We even included two-body phase spaces and archived all our results for some reason or another. This proved fortunate when, 5 years later, L.W. Jones proposed to compute large-angle elastic pp scattering and asked us whether we had any possibility to do so. The idea was that there might be a statistical intermediate state decaying into two protons isotropically. The cross-section for this process (visible, if existing, only far outside the diffraction region) should be proportional to the ratio

$$
\left.\frac{\mathrm{d} \sigma}{\mathrm{d} E}\right|_{q_{0}} \sim \frac{2 \text {-body phase space }}{\sum_{n} n \text {-body phase spaces }} .
$$

We dug out our old numerical results and found [4] an energy dependence $\sim \exp \left(-3.3 E_{\mathrm{cm}}[\mathrm{GeV}]\right)$, in agreement with experiments. This numerical result could, in simple analytical models (equal particles) only be reproduced if one suppressed there the factor $1 / N$ ! in front of the phase space integrals, otherwise one would obtain $\exp \left(-\right.$ const. $\left.E^{\alpha}\right)$ with $\alpha<1$ [5-7]. But omitting $1 / N$ ! amounted to considering the particles to be distinguishable - a most important observation which triggered all the rest, but which was greeted with pitiful smiles by those to whom I talked about it, although I tried to argue that indeed the particles should be effectively distinguishable, since the factor was $1 / N$ ! only for like particles, whereas when there were several different kinds, then

$$
\frac{1}{N !} \int \ldots \rightarrow \frac{1}{\prod_{i} N_{i} !} \int \ldots,
$$

where $i$ goes over all types of particles. We had in our earlier numerical computations used some 40 or 50 different types of particles (including some resonances and counting each state: spin, isospin, antiparticle, etc., as a new kind), and it so happened that, on the average, all $\left\langle N_{i}\right\rangle \leq 1$, so that the main contributions to Eq. (25.1) had come from integrals whose factor $1 / \prod_{i} N_{i} !=1$. We then worked out a model of massless distinguishable particles [5] which was so simple that I present it here.

Let $N$ massless, distinguishable particles be enclosed in a box of volume $V$ and let $\epsilon_{1}, \epsilon_{2}, \ldots, \epsilon_{i}, \ldots$ denote the single-particle energy levels with occupation numbers 
$n_{1}, n_{2}, \ldots, n_{i}, \ldots$. Then there will be

$$
\frac{N !}{n_{1} ! n_{2} ! \ldots} \text { states of total energy } E=\sum n_{i} \epsilon_{i}, \quad N=\sum n_{i} .
$$

If particles can be freely created, the partition function will be

$$
Z=\sum_{N=0}^{\infty} \sum_{\sum n_{i}=N} \frac{N !}{\prod_{i} n_{i} !} \exp \left(-\frac{1}{T} \sum n_{i} \epsilon_{i}\right)=\sum_{N=0}^{\infty}\left[\sum_{i} \exp \left(-\frac{\epsilon_{i}}{T}\right)\right]^{N}
$$

Replacing as usual

$$
\exp \left(-\frac{\epsilon_{i}}{T}\right) \longrightarrow \frac{V}{2 \pi^{2}} \int p^{2} \exp \left(-\frac{p}{T}\right) \mathrm{d} p=\frac{V T^{3}}{\pi^{2}},
$$

we find

$$
Z=\sum_{N=0}^{\infty}\left(\frac{V T^{3}}{\pi^{2}}\right)^{N}=\frac{1}{1-V T^{3} / \pi^{2}}
$$

which has a pole at $T_{\mathrm{c}}=\left(\pi^{2} / V\right)^{1 / 3}$, where the energy density would also diverge, implying that $T_{\mathrm{c}}$ is the maximum temperature for this system. Now comes the miracle: putting $V=V_{\text {hadron }}=(4 \pi / 3)\left(1 / m_{\pi}\right)^{3}$ gives $T_{\mathrm{c}} \approx 185 \mathrm{MeV}$, very near a 'temperature' that has been familiar for many years from cosmic ray physics $\left(p_{\perp}\right.$ distribution).

\subsection{Early Bootstrap}

Quite apart from hadrons not being massless, the above model is pure cheating if you only look at it a little closer. But it had enough cheating power to convince me that something in it must be true. The next move had thus to be to build a better model with real, massive, hadrons while keeping the idea of many different types of them being involved.

For the sake of clarity, I present now a simplified version (Boltzmann statistics, non-relativistic approximation) of the original paper [8]. Consider a mixture of ideal gases, each gas belonging to one species of particle with mass $m_{i}$; all particles can be freely created and absorbed. The total partition function is the product of the partition functions of the individual gases labelled by $i$. We have

$$
\begin{aligned}
\ln Z_{i}(T, V) & =\frac{V}{(2 \pi)^{3}} \int \mathrm{e}^{-\sqrt{p^{2}+m_{i}^{2}} / T} \mathrm{~d}^{3} p=\frac{V T}{2 \pi^{2}} m_{i}^{2} K_{2}\left(m_{i} / T\right) \\
& \approx V\left(\frac{T}{2 \pi}\right)^{3 / 2} m_{i}^{3 / 2} \mathrm{e}^{-m_{i} / T}
\end{aligned}
$$


Thus in this approximation,

$$
\ln Z=\sum_{i} \ln Z_{i}=V\left(\frac{T}{2 \pi}\right)^{3 / 2} \sum_{i} m_{i}^{3 / 2} \mathrm{e}^{-m_{i} / T},
$$

where the sum over $i$ goes over all hadron species which can in principle participate, including resonances and counting each spin and isospin state separately. Assuming most possible particles to be as yet unknown, it is convenient to introduce the mass spectrum $\rho(m)$, where

$$
\rho(m) \mathrm{d} m=\text { number of different hadron states in }\{m, \mathrm{~d} m\},
$$

which, of course, is also unknown (except for a few $\delta$-functions at low-lying stable masses and Breit-Wigner functions at the established resonances). We then replace the sum over $i$ by an integral over $\rho(m)$ and obtain

$$
Z(T, V)=\exp \left[V\left(\frac{T}{2 \pi}\right)^{3 / 2} \int_{0}^{\infty} \rho(m) m^{3 / 2} \mathrm{e}^{-m / T} \mathrm{~d} m\right]
$$

For the exact relativistic expression with Fermi and Bose statistics, see [8].

What now? We have a formula which tells us exactly nothing as long as $\rho(m)$, i.e., the complete $\rho(m)$, is not given. Here comes the key idea: statistical bootstrap.

\section{The Bootstrap Idea}

We can present this in four steps:

- We are after the description of 'fireballs', i.e., highly excited and decaying hadronic systems of hadronic size $V_{0}$, composed of hadrons. Our earliest results (phase space, large-angle elastic scattering) had suggested that the set of constituent hadrons should also include all resonances, which therefore must be counted in $\rho(m)$.

- The hadronic system of size $V_{0}$ (fireball) is, however, itself a highly excited hadron and is therefore not essentially different from a resonance: there should be just one set of hadrons, viz.,

$$
\{\pi, \mathrm{K}, \text { resonances, } \mathrm{N}, \Lambda, \Sigma \text {, more resonances, } \ldots, \text { fireballs ad infinitum }\},
$$

and all should appear in $\rho(m)$.

- The partition function Eq. (25.10) can be written down, using the density of states of the system it describes (a fireball if $V \approx V_{0}$ is the hadron volume!):

$$
Z\left(T, V_{0}\right)=\int_{0}^{\infty} \sigma\left(V_{0}, m\right) \mathrm{e}^{-m / T} \mathrm{~d} m
$$


where $\sigma\left(V_{0}, m\right)$ is the density of states of the fireball at mass $m$.

- Since $\rho(m)$ should include fireballs and since $\rho$ and $\sigma$ are densities of states of essentially the same objects, the two functions should be essentially the same, at least for large $m$. A weak requirement ('equal entropy') of this sort was

$$
\frac{\log \rho(m)}{\log \sigma\left(V_{0}, m\right)} \underset{m \rightarrow \infty}{\longrightarrow} 1 \text {. }
$$

On the other hand, one has the identity of Eqs. (25.10) and (25.11):

$$
\int_{0}^{\infty} \sigma\left(V_{0}, m\right) \mathrm{e}^{-m / T} \mathrm{~d} m=\exp \left[V\left(\frac{T}{2 \pi}\right)^{3 / 2} \int_{0}^{\infty} \rho(m) m^{3 / 2} \mathrm{e}^{-m / T} \mathrm{~d} m\right]_{(25}
$$

The two Eqs. (25.12) and (25.13) together form the bootstrap condition. In words, fireballs

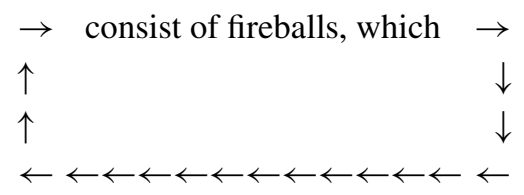

\section{Consequences}

From the bootstrap condition, it can be proved that the mass spectrum must grow exponentially (for large $m$ ):

$$
\rho(m) \underset{m \rightarrow \infty}{\longrightarrow} \frac{\text { const. }}{m^{a}} \mathrm{e}^{+m / T_{0}} .
$$

Together with its low mass (empirical) part, this then constituted the so far unknown mass spectrum $\rho(m)$.

Inserting this into (25.10) shows that the integral does not converge if $T>T_{0}$ : the partition function has a singularity at $T_{0}$ whose nature depends on the value of $a$. Thus there were three predictions:

- for sufficiently high collision energy, the $p_{\perp}$ distribution should be (very approximately $) \sim \exp \left(p_{\perp} / T\right)$ with $T \lesssim T_{0}$ [often called the $\exp \left(-6 p_{\perp}\right)$ law],

- the mass spectrum should grow exponentially,

- $T_{0}$ in the $p_{\perp}$ distribution and in the mass spectrum should be the same.

I presented these predictions and the whole model in a theory seminar in the fall of 1964. The result was disastrous. Nobody would believe it and I was shouted at: "but the mass spectrum does not grow exponentially". 
True enough, I had not yet even checked it. The next morning, I did so with beating heart and found what is depicted in Fig. 20.1 as the October 1964 situation. The $p_{\perp}$ distribution for $90^{\circ} \mathrm{C}$ elastic pp scattering [9] looked as in Fig. 17.4, where the slope required that $T_{0}=158 \mathrm{MeV}$, while for the mass spectrum, it was $T_{0}=$ $160 \pm 10 \mathrm{MeV}$. After the depressing seminar and an equally depressing night, this was such a relief that I never forgot this experience and henceforth was convinced that, all imperfections notwithstanding, the model was essentially right and that I should continue to work on it no matter what people would say (and they said a lot, mostly based on the misunderstanding that, in the model, a maximal temperature and/or an exponential spectrum were postulated, while these were in fact the results I was so proud of). The general rejection had one immense advantage: those few who worked with me (and myself) could proceed without fear of competition and without hurry.

After this anecdotal digression, consider now the energy density of such a system:

$$
\mathscr{E}=\frac{T^{2}}{V} \frac{\partial \ln Z}{\partial T}=\left(\frac{T}{2 \pi}\right)^{3 / 2}\left[\frac{3 T}{2} \int_{0}^{\infty} \rho(m) m^{3 / 2} \mathrm{e}^{-m / T} \mathrm{~d} m+\int_{0}^{\infty} \rho(m) m^{5 / 2} \mathrm{e}^{-m / T} \mathrm{~d} m\right] .
$$

With $\rho(m) \rightarrow$ const. $m^{-a} \exp \left(m / T_{0}\right)$ and at $T=T_{0}$, we find

$$
\mathscr{E}\left(T_{0}\right)=\text { const. }\left[\frac{3 T_{0}}{2}\left(m^{5 / 2-a}\right)_{M}^{\infty}+\left(m^{7 / 2-a}\right)_{M}^{\infty}+\text { finite parts }\right],
$$

where $M$ is assumed so large that the asymptotic expression Eq. (25.14) is valid for $m \geq M$.

The point I wish to make here is that, from Eq. (25.16), it follows that the energy density $\mathscr{E}$ is finite at $T_{0}$ if $a>7 / 2$ and infinite otherwise. This could have been interpreted as indicating that, since for $a>7 / 2$ one could reach the temperature $T_{0}$ at finite energy density, one could also pass over it into a region which would need another description. In other words, for $a>7 / 2$, there would be a phase transition, because all the usual features of a phase transition, in particular a critical temperature $T_{0}$, were present.

Unfortunately, and at that time for good reasons, I discarded this choice of $a$ and preferred $a=5 / 2$, giving infinite energy density at $T_{0}$, which therefore should be the highest temperature, approachable only asymptotically. And thus the discovery of the phase transition to the quark-gluon plasma, alas, escaped me (it was a bit early for that though!).

\section{Difficulties}

The model was still unsatisfactory in two respects:

- Inconsistency. While the fireball described by the partition function $Z\left(V_{0}, T\right)$ had a volume $V_{0}$, its constituents were treated as pointlike, although they were 
hadrons and fireballs, too. This inconsistency was not removed until 1978-1980. Then, however, with striking results (see below).

- Difficulty. No reason was given for treating resonances and fireballs as if they were particles. This obstacle was removed a few years later by using the arguments of Beth and Uhlenbeck [10] and Belenkij [11]. In fact these arguments put the somewhat shaky bootstrap idea (Sect.25.3) on rather firm ground and allowed us to state that a strongly interacting hadron gas can be described alternatively as a mixture of infinitely many ideal gases listed in the complete mass spectrum.

These arguments were given in extenso in three places and cannot be repeated here [12-14].

\section{Early Developments}

The model could not immediately be applied to hadron collisions, but it was important to do so in order to convince the non-believers (all theoreticians except for a set of measure zero) that it did work. Thus J. Ranft and myself worked out the so-called 'thermodynamical model' in which (see Fig. 25.1) collective velocity distributions were combined with bootstrap thermodynamics in local rest frames [15]. This mammoth paper of 141 pages was too long to be read by anybody, with the consequence that even today people rediscover (independently) results and techniques buried in it. For us, it was a kind of handbook used in further work on the model. In particular, J. and G. Ranft and their collaborators in Leipzig developed it and confronted it with experiment (see later). We produced an 'Atlas of Particle Spectra' (Fig. 25.2) [16], in which we reproduced measured momentum spectra and predicted unmeasured ones up to $p_{0}=800 \mathrm{GeV} / \mathrm{c}$ [for p-nucleus ( $\left.\mathrm{Be}, \mathrm{Al}, \mathrm{Cu}, \mathrm{Pb}\right)$ up to $70 \mathrm{GeV} / \mathrm{c}$ ] and discovered that the model obeyed 'Feynman scaling' and 'limiting fragmentation' [12].

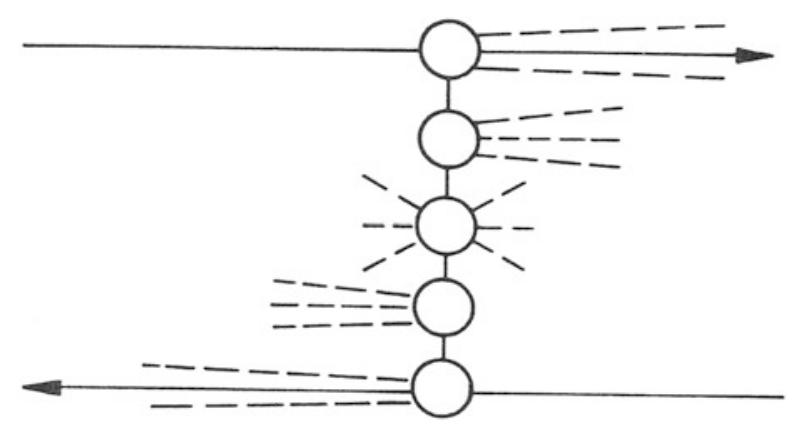

Fig. 25.1 Bootstrap thermodynamics is applied to each bubble in its rest frame. Lorentz transformation to the laboratory (or centre of mass) frame and integration over all possible impact parameters and velocities gives realistic predictions of particle momentum spectra 

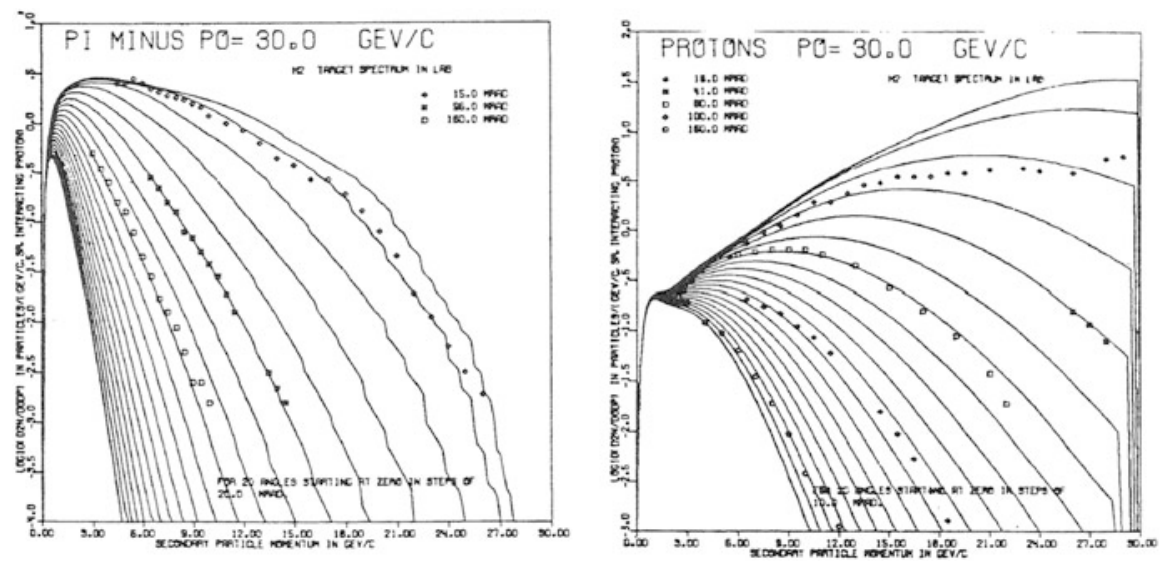

Fig. 25.2 Predicted and measured momentum spectra of $\pi^{-}$and p. Figures taken from the 'Atlas of Particle Spectra' [16]. On left the $\pi^{-}$-angular spectra computed starting at zero (upper right) in steps of $20 \mathrm{mrad}$, data are for 15,96 , and $160 \mathrm{mrad}$. On right the p-angular spectra computed starting at zero (upper right) in steps of $10 \mathrm{mrad}$, data are for $18,41,60,100$, and $160 \mathrm{mrad}$

While all these years much talk was about quarks, but experimental searches did not reveal any; confinement and QCD were still far in the future. It was speculated that maybe quarks are so heavy that they would be practically unobservable. Bootstrap thermodynamics was presumably the best way to calculate $q \bar{q}$ production rates as a function of their mass (applying perturbation theory to this problem was a then fashionable nonsense). The model was used to predict [17] $K \bar{K}, p \bar{p}$, d $\bar{d}$, and $\mathrm{q} \overline{\mathrm{q}}$ pair production with the result that the production rates of the first three agreed with experiment and that for quarks a mass of 4-5 nucleon masses would make them practically unobservable (with the techniques known at that time), even if they could, in principle, exist as free particles. If anyone had suggested to me then a $q \bar{q}$ plasma, I would have declared it impossible (alas, again).

One often-heard objection to the model was that resonances (inside a fireball) would not live long enough to justify treating them as 'particles'. Matthiae [18] proved, using the principle of detailed balance, that they might just live long enough.

It was tempting to apply the model to astrophysics: the Big Bang and neutron stars [19]. This triggered a number of papers, notably the one by Huang and Weinberg [20] and by Wheeler [21]. The then proposed 'limited temperature $\left(T_{0}\right)$ Big Bang' depended entirely on the (at that time reasonable) interpretation of the model as yielding an infinite energy density at $T_{0}$.

For an easily readable summary of the situation up to 1972, see [14], where no prior knowledge about statistical bootstrap is required. 


\section{Microcanonical Bootstrap}

The invention of Veneziano's dual resonance model and the discovery that it also predicted an exponential mass spectrum [22, 23] added to the credibility the statistical bootstrap model had won in the meantime by successful applications. A number of people became interested and the following few years saw the appearance of some important papers.

S. Frautschi, who also coined the name 'Statistical Bootstrap Model' (SBM) [24], made a great breakthrough by reformulating the model (grand) microcanonically, instead of (grand) canonically. (Roughly, the original model was the Laplace transform of Frautschi's.) His bootstrap equation (BE) reads in invariant notation [25]

$$
H \tau\left(p^{2}\right)=H \sum_{m_{\text {in }}} \delta_{0}\left(p^{2}-m_{\text {in }}^{2}\right)+\sum_{n=2}^{\infty} \frac{1}{n !} \int \delta^{4}\left(p-\sum_{i=1}^{n} p_{i}\right) \prod_{i=1}^{n} H \tau\left(p_{i}^{2}\right) \mathrm{d}^{4} p_{i},
$$

where $\tau\left(m^{2}\right) \mathrm{d} m^{2} \equiv \rho(m) \mathrm{d} m, H$ is a universal constant, and $m_{\text {in }}$ runs over the low mass stable hadrons (often only the pion). The philosophy was the same as before (with slight differences): $\tau\left(p^{2}\right)$ on the left-hand side of Eq. (25.17) was the density of states of the fireball with mass $m=\sqrt{p_{\mu} p^{\mu}}$ to be described, while the right-hand side expressed that it was either just one of the input particles or else composed of particles $i$ having mass spectra $\tau\left(p_{i}^{2}\right)$.

Frautschi, Hamer, and Carlitz [24, 26-30] drew many important conclusions which would have been impossible to draw in the earlier canonical language. The new language used a vocabulary familiar to particle physicists (phase space), while the canonical one was repellent to them at that time. (Today, knowledge of thermodynamics is more widespread among high energy physicists.) Some of the new results were:

- Fireballs would predominantly decay into two fragments, one heavy and one light.

- By iterating their BE with realistic input, they found numerically $T_{0} \approx 140 \mathrm{MeV}$ and $a=2.9 \pm 0.1$ (which ruled out the until then adopted value $a=5 / 2$ ).

- Each imposed conservation law implemented by fixing a quantum number, e.g., baryon number $\rho(B, m)$, in the mass spectrum, increases the value of $a$ by $1 / 2$.

- The microcanonical description gives a detailed account of what happens near $T_{0}$ (clustering, overshooting $T_{0}$, etc.).

Nahm [31] had meanwhile proved analytically that $a$ must be exactly 3 . This and the Frautschi-Hamer numerical result strengthened everyone's belief that $T_{0}$ must be a limiting temperature, since the energy density tends to infinity when $T \rightarrow T_{0}$. Thus, there was no indication of a phase transition to a new state of matter (alas, once more).

More checks with experiments were made ( $\mathrm{p} \overline{\mathrm{p}}$ annihilation, pair production, correlations, heavy particle production, ISR secondary spectra, Ericson fluctuations 
[32], and so on), which cannot be reviewed here. Instead I refer to the excellent review papers by Ilgenfritz, Kripfganz, and Möhring [33] and by Tounsi [34], in which the current technical improvements of the model (carried out in Leipzig, CERN, and Paris) are also described.

During these years, a group around L. Sertorio was busy relating the SBM to $S$ matrix theory [35-37] and clearing up many of the main open questions. This work is so extensive that I must refer to Sertorio's very complete and important review paper [38], which gives a full account of the more theoretical aspects up to 1978 .

Other work included attempts, some successful, some less so, to equip the model with the following:

- Bose and Fermi statistics (present in the original canonical formulation [8], but extremely difficult to formulate in Frautschi's microcanonical one); Bose statistics was achieved [39-42], not Fermi.

- Internal symmetries [charge, isospin, SU(3), etc.] using group theoretical methods (based on early pioneering work by Cerulus [43]) [44-50].

- Angular momentum conservation [51, 52].

Much attention was given to the relation between SBM and dual resonance, string, and Regge pole models with the result that there was indeed a close resemblance, but not identity of the models [53-57]. Related to this question was a reconsideration of large-angle elastic scattering and Ericson fluctuations [32, 58-63].

Nucleon-antinucleon annihilation received new interest [64-68].

The description of particle production (momentum spectra, production rates, and so on) was improved [69-75] and extended to non-symmetrical collisions ( $\pi \mathrm{N}, \mathrm{KN}$, etc.) [76] and even to p-nucleus collisions [77].

\section{Exact Analytical Solutions of the BE}

During the same years, our analytical understanding of the BE Eq. (25.17) advanced and solutions were constructed. The pioneer was J. Yellin, who had proposed Eq. (25.17) and solved it formally by Fourier transform (strictly speaking, not existing) and, via Eq. (25.22), by expansion in terms of phase space integrals [25]. This triggered a series of papers in which the BE was solved by Laplace transform (which does exist). The decay integral equation (sum over all possible decay modes) and its identity with the BE were discovered and a number of formal properties derived [45, 78-84]. Even a second quantized SBM was constructed, which led to the strange discovery that the creation and destruction operators of hadron clusters obey a non-associative (and, of course, non-commutative) algebra [85]. 


\section{The Bootstrap Function}

The analytical solution of the BE via Laplace transform mentioned above was so important for further development that I present it here. It goes as follows. Define the Laplace transforms

$$
\begin{gathered}
\Phi(\beta):=\int \mathrm{e}^{-\beta_{\mu} p^{\mu}} H \tau\left(p^{2}\right) \mathrm{d}^{4} p, \\
\varphi(\beta):=\int \mathrm{e}^{-\beta_{\mu} p^{\mu}} H \delta_{0}\left(p^{2}-m_{\mathrm{in}}^{2}\right) \mathrm{d}^{4} p,
\end{gathered}
$$

where $\left(\beta_{\mu} \beta^{\mu}\right)^{-1 / 2}=T$ is the temperature in the rest frame of the system. Laplace transforming Eq. (25.17) then gives

$$
\Phi(\beta):=\varphi(\beta)+\mathrm{e}^{\Phi(\beta)}-\Phi(\beta)-1 .
$$

Writing

$$
G(\varphi):=\Phi(\beta),
$$

this yields the equation

$$
\varphi=2 G-\mathrm{e}^{G}+1
$$

which has been solved for the 'bootstrap function' $G(\varphi)$ by power expansion [25] and by an integral representation exploiting the analytical structure of Eq. (25.22) [86]. Most illuminating is the graphical solution of Eq. (25.22): draw the curve $x=$ $2 y-\mathrm{e}^{y}+1$ on left in Fig. 17.5 and interpret it as the graph of the inverse function, on right in Fig. 17.5.

It follows that, for physical systems,

$$
\begin{gathered}
\varphi \leq \varphi_{0}=\ln 4-1, \\
\Phi \leq \Phi_{0}=\ln 2 .
\end{gathered}
$$

In the simple case of one input mass, we have from Eq. (25.19)

$$
\varphi(\beta)=2 \pi H m_{\mathrm{in}}^{2} \frac{K_{1}\left(\beta m_{\mathrm{in}}\right)}{\beta m_{\mathrm{in}}},
$$

and the condition Eq. (25.23) reads

$$
2 \pi H m_{\mathrm{in}}^{2} \frac{K_{1}\left(\beta_{0} m_{\mathrm{in}}\right)}{\beta_{0} m_{\mathrm{in}}}=\ln 4-1,
$$


which fixes a maximal temperature $T_{0}=1 / \beta_{0} \approx m_{\mathrm{in}}$. Thus, in this version of the model, $T_{0}$ is calculable (depending on $H$ and $m_{\text {in }}$ ).

Furthermore, one recovers the earlier result of Nahm [31] that the singularity of $G(\varphi)$ at $\varphi_{0}$ is of the square-root type: $G(\varphi)=\Phi_{0}-$ const. $\sqrt{\varphi_{0}-\varphi}+\cdots$, from which it follows by inverse Laplace transformation of Eq. (25.18) that $\tau\left(m^{2}\right) \sim$ const. $m^{-3} \exp \left(m / T_{0}\right)$ [80], confirming once more that the energy density at $T_{0}$ is infinite and hence $T_{0}$ is a limiting temperature which might be attained or even exceeded only in transient states of superheating, but never in true equilibrium: so still no phase transition to a new regime (alas, for the fourth time).

It is important to observe that the same Eq.(25.22) is obtained independently of:

- the number of spacetime dimensions [83],

- the number of input particles (trivial),

- Abelian or non-Abelian symmetry constraints [50].

What changes is only the function $\varphi$, which may become very complicated, depending on various input masses and chemical potentials besides the temperature, while the relation between $\Phi$ and $\varphi$ is always given by Eq. (25.22). Therefore, the 'bootstrap function' $G(\varphi)$ has a sort of invariant significance for SBM and it will also govern the discussion of the phase transition to which we will eventually come. [Note, however, that changing the BE by truncating it $[80,87,88$ ] or by making the volume $n$-dependent [89] will also change Eq. (25.22).] This invariance of the Laplace transformed BE suggests that it may appear in other contexts, and indeed it does: in renormalization theory, in nonlinear differential equations, and in combinatorics (references given in [86]). In this latter field, Eq. (25.22) was already discovered in the last century by Schröder [90]. He was solving a combinatorial problem which he explained in the following words (free translation):

Given $n$ elements, e.g., material points freely movable in space, one divides the set of $n$ elements arbitrarily into subsets; some possibly containing only one element, some with two, three, etc., elements. Each set of more than one element is to be surrounded by a closed surface, which, together with its content, shall be considered as a new element on an equal footing with all the others. This new set of (obviously fewer than $n$ ) elements is submitted to the same procedure as long as one likes or until it ends because the next step would result in a single element. We wish to find the number of different complexions which can be generated by this procedure.

This is indeed SBM in its most abstract form. He terminates his paper with the prophetic words:

Considerably more difficult would be the solution of the above problems in the case where, among the original elements, several are identical.

As if he had already known that, more than 100 years later, we would have difficulties with Bose and Fermi statistics! 


\section{The State of Affairs up to 1978}

The bootstrap equation (various versions) had been solved and the ensuing thermodynamics had been applied in particle physics, astrophysics, and cosmology. For particle physics applications, further assumptions about collective motions had been necessary [15], assumptions that had nothing to do with bootstrap thermodynamics, but contributed to the successes of the model. These and the confirmations coming from the dual models strengthened the belief that the model was basically right. The only challenge, large $p_{\perp}$, was interpreted as (at least partly) due to pre-equilibrium processes $[15,91]$ and/or angular momentum [52].

The picture was then as follows:

- Fireballs consist of fireballs (still pointlike, which is inconsistent, but bothered nobody).

- As a consequence, there is a limiting temperature $T_{0}$ which cannot be reached at finite energy density.

This picture seemed to provide a non-perturbative, analytically soluble model of the many-body aspects of strong interactions, running against a 'phase transition' whose other side would, however, be a forbidden land. It turned out later that removing the inconsistency of pointlike constituent fireballs would drastically change the situation and make the 'phase transition' a true transition with the other side no longer forbidden.

The state of affairs through 1978 is reviewed in the articles:

- Hagedorn [14] of 1973 for a heuristic introduction, ideology, and general results;

- Tounsi [34] of 1973 and Ilgenfritz et al. [33] of 1977 for a critical discussion and confrontation with experiment, and

- Sertorio [38] of 1978 for more formal questions, the relation to the $S$-matrix, and so on.

Each of these needs the others for complementation.

\subsection{The Phase Transition: Hadron Matter-Quark Matter}

A phase transition has two sides. In our particular case, only lattice-QCD is so far able to handle both sides simultaneously; all other approaches (including ours) treat them separately and join them in hybrid models. That a quark-gluon matter phase might exist has been speculated since as early as 1969 [92] (maybe earlier ${ }^{1}$ ) and the first theoretical paper really devoted to it seems to be Carruthers' in 1973 [93]. From

\footnotetext{
${ }^{1}$ I have reviewed Ref. [92] and find no mention of quark or parton phase, or for that matter, phase transition in this well-known publication. The very first mention of matter made of quarks that I know today about is by Ivanenko and Kurdgelaidze in 1965, see Ref. [8] in Chap. 11 on page 91. (JR).
} 
about 1975 on, so many papers appeared that I must refer to the articles collected in the Bielefeld proceedings of 1980 and 1982 [94, 95], where references are given.

In the present context, the approach from the hadron side is of interest. The obvious argument-who did invent it?-would be: if you compress hadron matter sufficiently, hadrons will overlap and cease to exist as such. You then have a quarkgluon soup. The ideas was used in many papers and got much support from the MIT bags [96], which are quark-gluon matter inside, hadrons outside. As many of the properties of bags strangely resemble the clusters (formerly called fireballs) of the SBM, one might expect that bootstrap thermodynamics would be equivalent to the thermodynamics of a bag gas. Indeed, an explicit model constructed by Baacke [97] made a phase transition very likely to happen near $T_{0}$. Two years earlier, Cabibbo and Parisi [98] had already proposed that the singularity found in SBM at $T_{0}$ should be related to the transition to the quark-gluon phase. These two arguments supported each other beautifully: SBM would provide for the singularity necessary for a true phase transition, while the bag gas model produced the crossing of the pressure curves of the plasma and the hadron gas near $T_{0}$.

Unfortunately, SBM was still in an underdeveloped stage in which two arguments spoke against Cabibbo and Parisi's interpretation:

- The energy density $\mathscr{E} \rightarrow \infty$ when $T \rightarrow T_{0}$.

- Quarks and gluons did not appear anywhere in SBM.

Therefore, how could a singularity, which could not be reached at finite energy density, indicate a transition to a phase whose constituents did not appear in the model? I felt strongly this way.

Some people, however, felt-rightly - that these objections would be overcome by technical development without touching the essential features of SBM. Thus a number of papers, originating mostly in the fertile and critical soil of Bielefeld, investigated the various aspects of a phase transition, in the presence of an exponential spectrum: critical exponents, influence of fugacities, types of transition, and others [81, 99-106], to mention only a few references.

At this time, W. Nahm, during a workshop at Erice [107], severely criticized the shortcomings of the model and pointed out that they would forbid its application in astrophysics and to phase transitions, while particle physics applications might still be reasonable [108]. His criticism coincided, however, with a new formulation of SBM, just presented at the same workshop, in which most of the inconsistencies were removed [109], and to which we now turn.

\section{Hadron Volumes}

The breakthrough came when J. Rafelski and myself tried to apply SBM to heavy ion collisions. There it was obvious that we could no longer deal with pointlike 'constituent clusters' of the extended clusters to be described. It took us a considerable time to equip the model with baryon conservation and proper hadron 
volumes. Our efforts, helped in a later stage by I. Montvay, resulted in the following treatment of the volume question:

- In the fully relativistic formulation we strived for, the volume had to be a fourvector parallel to the system it confined [110]:

$$
V \longrightarrow V^{\mu}=A(m, \ldots) p^{\mu}
$$

- If a cluster c consisted of clusters $i$, then

$$
V_{\mathrm{c}}^{\mu}=K \sum_{i} V_{i}^{\mu}
$$

where $K$ is some constant.

- Hence, with Eq. (25.26),

$$
A\left(m_{\mathrm{c}}, \ldots\right) p_{\mathrm{c}}^{\mu}=K \sum_{i} A\left(m_{i}, \ldots\right) p_{i}^{\mu}
$$

- Since momentum conservation requires $p_{\mathrm{c}}^{\mu}=\sum p_{i}^{\mu}$, it follows that the volume is proportional to the mass:

$$
K=1 \quad \text { (dense packing) }, \quad A=\text { const. (independent of } m, \ldots) \equiv 1 / 4 \mathscr{B} \text {. }
$$

We identify $\mathscr{B}$ with the bag constant. These and other results were presented in Erice in the 1978 Workshop on Hadronic Matter at Extreme Energy Density [109]. However, this paper leaves one question unsolved: after treating the volume correctly on the level of the BE, the hadron gas should also take particle volumes into account (as already done by Baacke [97]). We later succeeded in doing so [111], by introducing the notion of the 'available volume', defined by subtracting (cluster) volumes from the 'external' (confining box) volume:

$$
\Delta^{\mu}:=V_{\mathrm{ext}}^{\mu}-\sum_{\text {all particles } i} V_{i}^{\mu} .
$$

Then, instead of keeping (as usual) the external volume fixed, we required $\Delta$ to be constant when summing over particle numbers and integrating over the mass spectrum, whereby $V_{\mathrm{ext}}^{\mu}$ was pushed to infinity. By this trick, the partition function became formally that of pointlike particles in the available volume $\Delta$, which we knew how to calculate explicitly. Thereafter, $\Delta$ had to be eliminated by using

$$
\left\langle V_{\mathrm{ext}}\right\rangle=\Delta+\frac{\langle E\rangle}{4 \mathscr{B}} .
$$


Then the energy density (and all other physical quantities) could be calculated. It turned out that

$$
\frac{\langle E\rangle}{\left\langle V_{\text {ext }}\right\rangle}=\mathscr{E}(T, \mu)=\frac{\mathscr{E}_{\mathrm{pt}}(T, \mu)}{1+\mathscr{E}_{\mathrm{pt}}(T, \mu) / 4 \mathscr{B}},
$$

and similarly for other quantities. Here $\mathscr{E}_{\mathrm{pt}}$ is the fictitious 'point particle energy density', which we can explicitly calculate and which diverges on the 'critical curve' [given by an equation like Eq. (25.25) with the baryon chemical potential $\mu$ incorporated]. Thus on the critical curve, the energy density is now finite [Eq. (25.32) with $\left.\mathscr{E}_{\mathrm{pt}}(T, \mu) \rightarrow \infty\right]$ :

$$
\mathscr{E}\left(T_{\text {crit }}, \mu_{\text {crit }}\right)=4 \mathscr{B}=\text { bag energy density . }
$$

The baryon number density is also finite along this curve, while the average cluster mass and volume tend to infinity, and the pressure to zero: everything coalesces into one infinite cluster with the energy density of a bag. Since inside highly excited (large) bags we have a quark-gluon gas, we should identify the single infinite cluster with quark-gluon matter, the transition to which is achieved just on the critical curve.

We have finally arrived at a sophisticated, relativistic van der Waals-type gas of strongly interacting particles: finite (mass proportional) cluster volumes represent repulsive forces, while the exponential mass spectrum resulting from bootstrap represents the attractive forces. The thus completed SBM leads to a phase transition on a critical curve (Fig. 25.3) qualitatively common to many models [95, 106, 112116] to mention only a few of the many references. The results of QCD lattice calculations (see [95]) support our conclusions.

Yet, one might argue, how do you know that the singularity indicates a phase transition to quark-gluon matter? Nowhere in the SBM do quarks appear as input particles! Short of a proof, I can at least propose a chain of heuristic arguments:

- The identical properties of QCD bags and SBM clusters: both have the same mass-volume relation and the same mass spectrum up to the finest details with respect to conservation laws $[117,118]$. These identities lead to the conclusion that QCD bags of interacting fields and SBM clusters are complementary descriptions of the same objects below the transition.

- If we are thus free to claim that, inside our clusters we do indeed have quarkgluon matter (though correlated by colour neutrality of constituent subclusters), then we do indeed implicitly have quarks and gluons in the model.

- The very nature of the bootstrap approach makes it rather irrelevant which are the input particles, provided the basic quantum numbers are represented. Instead of starting with quarks and gluons and imposing their interaction and confinement to colourless bound states, we have accepted nature's own solution of the $Q C D$ bound state problem and used the $\pi$ and the nucleon (others could be added) as input. (After all, to describe a helium gas, you do not start with neutrons, protons, and electrons!)

Thus, without having a formal proof, I believe one can justify asserting that SBM predicts a phase transition (of which not yet all details are known) to a quark- 
Fig. 25.3 The singular curve of the statistical bootstrap model. In the shaded region, the model is unreliable, because there the (otherwise negligible) effects of Bose-Einstein and Fermi-Dirac statistics become important

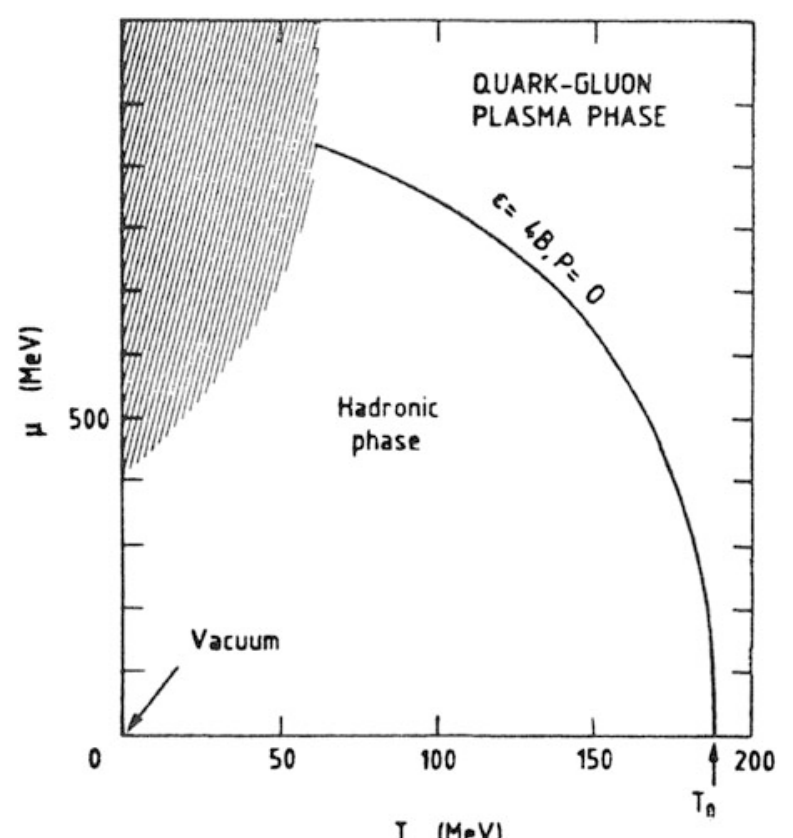

gluon matter phase, as J. Rafelski and I proposed at the 1980 Bielefeld symposium $^{2}$ $[119,120]$.

A further criticism was brought up by V.V. Dixit and H. Satz: in the standard thermodynamical limit, a hard sphere gas, even with an exponential mass spectrum, cannot produce a singularity at any finite temperature [121]. Therefore, our method with the available volume violates the standard rules of taking the thermodynamic limit. The question was discussed at length [122] and it was shown that, since hadron matter at the regime near transition cannot be enclosed in boxes of fixed volume, the standard thermodynamical limit is not suitable. Using the 'grand canonical pressure ensemble' (without recourse to the available volume technique), the result of Dixit and Satz was reproduced, (see Chap. 24), our old results were also recovered, and this settled the question.

Here I must stop. Work goes on. For a review of present developments, applications, problems, and progress, see Rafelski and Danos [123, 124], also [125], and a rather daring and controversial paper of mine [91].

And if you ask me now why it took 27 years to arrive at the present (still problematic) state, let me answer with Shakespeare [126]:

There are more things in Heaven and Earth, Horatio, than are dreamt of in your philosophy.

\footnotetext{
${ }^{2}$ Two months later a workshop at GSI was held in October 1980, and in this volume we reprint these two contributions in Chaps. 26 and 27.
} 
Acknowledgements My sincere thanks and admiration go to all who have worked in this field and brought it into the present state, but in particular to those with whom I had, over the years, the pleasure of close contact, discussions, and collaboration: A. Auberson, R.D. Carlitz, F. Cerulus, M. Chaichian, J. Engels, B. Escoubès, E. Etim, G. Fast, R. Fiore, S. Frautschi, H. Grote, L.W. Jones, J. Kripfganz, J. Letessier, I. Montvay, W. Nahm, J. and G. Ranft, J. Rafelski, K. Redlich, H. Satz, K. Schilling, L. Sertorio, A. Tounsi, L. Turko, J. Vandermeulen, and U. Wambach.

Open Access This book is distributed under the terms of the Creative Commons Attribution Noncommercial License which permits any noncommercial use, distribution, and reproduction in any medium, provided the original author(s) and sources are credited.

\section{References}

1. H. Satz, Fortschr. Phys. 33, 259 (1985)

2. E. Fermi, Prog. Theor. Phys. 5, 570 (1950)

3. F. Cerulus, R. Hagedorn, Suppl. Nuovo Cimento 9, 646, 659 (1958)

4. G. Fast, R. Hagedorn, Nuovo Cimento 27, 208 (1963); G. Fast, R. Hagedorn, L.W. Jones, Nuovo Cimento 27, 856 (1963)

5. G. Auberson, B. Escoubès, Nuovo Cimento 36, 628 (1965)

6. H. Satz, Nuovo Cimento 37, 1407 (1965)

7. J. Vandermeulen, Bull. Soc. R. Sci. Lieège 46, 57 (1977)

8. R. Hagedorn, Suppl. Nuovo Cimento 3, 147 (1965)

9. J. Orear, Phys. Lett. 13, 190 (1964)

10. E. Beth, G.E. Uhlenbeck, Physica 4, 915 (1937)

11. S.Z. Belenkij, Nucl. Phys. 2, 259 (1956)

12. R. Hagedorn, Nucl. Phys. B 24, 93 (1970)

13. R. Hagedorn, CERN Yellow Report 71-12 (1971)

14. R. Hagedorn, in Cargèse Lectures in Physics, vol. 6, ed. by E. Schatz (Gordon \& Breach, New York, 1973), p. 643

15. R. Hagedorn, J. Ranft, Suppl. Nuovo Cimento 6, 169 (1968)

16. H. Grote, R. Hagedorn, J. Ranft, CERN Report (Black) (1970)

17. R. Hagedorn, Suppl. Nuovo Cimento 6, 311 (1968)

18. G. Matthiae, Nucl. Phys. B 7, 142 (1968)

19. R. Hagedorn, Astron. Astrophys. 5, 184 (1970)

20. K. Huang, S. Weinberg, Phys. Rev. Lett. 25, 895 (1970)

21. J.C. Wheeler, Astrophys. J. 169, 105 (1971)

22. S. Fubini, D. Gordon, G. Veneziano, Phys. Lett. B 29, 679 (1969)

23. S. Fubini, G. Veneziano, Nuovo Cimento A 64, 811 (1969)

24. S. Frautschi, Phys. Rev. D 3, 2821 (1971)

25. J. Yellin, Nucl. Phys. B 52, 583 (1973)

26. C.J. Hamer, S. Frautschi, Phys. Rev. D 4, 2125 (1971)

27. R.D. Carlitz, Phys. Rev. D 5, 3231 (1971)

28. S. Frautschi, Nuovo Cimento A 12, 133 (1972)

29. C.J. Hamer, Phys. Rev. D 10, 1458 (1974)

30. C.J. Hamer, Nucl. Phys. B 95, 173 (1975)

31. W. Nahm, Nucl. Phys. B 45, 525 (1972)

32. S.C. Frautschi, in Hadronic Matter at Extreme Energy Density, Erice, 1978, ed. by N. Cabibbo, L. Sertorio (Plenum Press, New York 1980), p. 1

33. E.M. Ilgenfritz, J. Kripfganz, H.J. Möhring, Fortschr. Phys. 25, 123 (1977)

34. A. Tounsi, Sixteenth Scottish Universities Summer School 1973, London, 1974

35. A. Bassetto, L. Sertorio, Nuovo Cimento A 14, 548 (1965)

36. P. Fré, L. Sertorio, Nuovo Cimento A 28, 538 (1975) 
37. P. Fré, Fortschr. Phys. 25, 579 (1977)

38. L. Sertorio, Riv. Nuovo Cimento 2, 1 (1979)

39. K. Fabricius, U. Wambach, Nucl. Phys. B 62, 212 (1973)

40. J. Engels, K. Fabricius, K. Schilling, Phys. Lett. B 59, 477 (1975)

41. J. Kripfganz, J. Ranft, Nucl. Phys. B 100, 302 (1975)

42. M. Chaichian, M. Hayashi, Phys. Rev. D 15, 402 (1977)

43. F. Cerulus, Nuovo Cimento 19, 528 (1961)

44. E.M. Ilgenfritz, J. Kripfganz, Nucl. Phys. B 62, 141 (1973)

45. C.J. Hamer, Phys. Rev. D 8, 3558 (1973)

46. J. Engels, K. Fabricius, K. Schilling, Phys. Lett. B 53, 65 (1974)

47. J. Engels, K. Fabricius, K. Schilling, Nuovo Cimento A 23, 581 (1974)

48. F. Csikor, I. Farkas, Z. Katona, I. Montvay, Nucl. Phys. B 74, 343 (1974)

49. J. Kripfganz, E.M. Ilgenfritz, Phys. Lett. B 48, 329 (1974)

50. K. Redlich, L. Turko, Z. Phys. B 92, 207 (1975)

51. C.B. Chiu, R.L. Heimann, Phys. Rev. D 4, 3184 (1971)

52. R. Hagedorn, U. Wambach, Nucl. Phys. B 123, 382 (1977)

53. M.I. Gorenstein, V.A. Miransky, V.P. Shelest, B.V. Struminsky, G.M. Zinovjev, Phys. Lett. B 43, 73 (1973)

54. H. Satz, Phys. Lett. B 44, 373 (1973)

55. J. Dethlefsen, H.B. Nielsen, H.C. Tze, Phys. Lett. B 48, 48 (1974)

56. M.I. Gorenstein, V.A. Miransky, V.P. Shelest, G.M. Zinovjev, H. Satz, Nucl. Phys. B 76, 453 (1974)

57. A. Strumia, G. Venturi, Lett. Nuovo Cimento 13, 337 (1975)

58. F. Johns, B. Margolis, W.J. Meggs, R.K. Logan, Phys. Rev. Lett. 29, 756 (1972)

59. G. Eilam, Y. Gell, B. Margolis, W.J. Meggs, Phys. Rev. B 8, 2871 (1973)

60. B. Margolis, W.J. Meggs, R.K. Logan, Phys. Rev. D 8, 199 (1973)

61. C.B. Chiu, Phys. Rev. D 13, 2091 (1976)

62. S. Kogitz, R.K. Logan, S. Tanaka, Phys. Rev. D 14, 1420 (1976)

63. R.K. Logan, M. Bates, S. Kogitz, Phys. Rev. D 25, 2441 (1982)

64. C.J. Hamer, Nuovo Cimento A 12, 162 (1972)

65. H.J. Möhring, J. Kripfganz, E.M. Ilgenfritz, J. Ranft, Nucl. Phys. B 85, 221 (1975)

66. H.J. Möhring, Nucl. Phys. B 87, 509 (1975)

67. J. Vandermeulen, Bull. Soc. R. Sci. Liège 46, 57 (1977)

68. H.J. Möhring, Acta Phys. Pol. B 9, 125 (1978)

69. J. Ranft, Phys. Lett. B 31, 529 (1970)

70. R. Hagedorn, J. Ranft, Nucl. Phys. B 48, 157 (1972)

71. J. Letessier, A. Tounsi, Nuovo Cimento A 11, 353 (1972)

72. J. Letessier, A. Tounsi, Nuovo Cimento A 13, 557 (1973)

73. J. Letessier, A. Tounsi, Nuovo Cimento A 15, 358 (1973)

74. J. Kripfganz, J. Ranft, Phys. Lett. B 55, 301 (1975)

75. J. Ranft, G. Ranft, Nucl. Phys. B 92, 207 (1975)

76. H.R. Gerhold, B. Buschbeck, Nuovo Cimento A 22, 39 (1974)

77. J.P. Alard, A. Baldit, R. Brun, J.P. Costilhes, J. Dhermain, J. Fargaix, L. Fraysse, J. Pellet, G. Roche, J.C. Tamain, A. Cordaillat, A. Pasinetti, Nuovo Cimento A 30, 320 (1975)

78. E.M. Ilgenfritz, J. Kripfganz, Nucl. Phys. B 56, 241 (1973)

79. I. Montvay, Phys. Lett. B 42, 466 (1972)

80. R. Hagedorn, I. Montvay, Nucl. Phys. B 59, 45 (1973)

81. R. Fiore, R. Page, L. Sertorio, Nuovo Cimento A 37, 45 (1977)

82. M. Feigenbaum, L. Sertorio, Nuovo Cimento A 43, 31 (1978)

83. H. Satz, Phys. Rev. D 20, 582 (1979)

84. R. Fiore, Z. Phys. C 4, 281 (1980)

85. E. Etim, R. Hagedorn, Nucl. Phys. B 131, 429 (1977)

86. R. Hagedorn, J. Rafelski, Commun. Math. Phys. 83, 563 (1982)

87. C.B. Chiu, J. Johnson, Phys. Lett. B 42, 475 (1972)

88. C.B. Chiu, Nucl. Phys. B 54, 170 (1973) 
89. I.Ya. Pomeranchuk, Dokl. Akad. Nauk. 78, 889 (1951)

90. E. Schröder, Z. Math. Phys. 15, 361 (1870) (For a modern representation of this work, see L. Comtet, Advanced Combinatorics, Reidel Publishing Co., Dordrecht, 1970)

91. R. Hagedorn, Riv. Nuovo Cimento 6, 1 (1984)

92. J.D. Bjorken, E. Paschos, Phys. Rev. 185, 1975 (1969)

93. P. Carruthers, Collect. Phenom. 1, 147 (1973)

94. H. Satz (ed.), Statistical Mechanics of Quarks and Hadrons, Bielefeld Symposium 1980 (North Holland, Amsterdam, 1980)

95. M. Jacob, H. Satz (eds.), Quark Matter Formation and Heavy Ion Collisions, Bielefeld Workshop 1982 (World Scientific, Singapore, 1982)

96. A. Chodos, R.L. Jaffe, K. Johnson, C.B. Thorn, V.F. Weisskopf, Phys. Rev. D 19, 3471 (1974)

97. J. Baacke, Acta Phys. Pol. B 8, 625 (1977)

98. N. Cabibbo, G. Parisi, Phys. Lett. B 59, 67 (1975)

99. D.E. Miller, Phys. Rev. Lett. B 64, 193 (1976)

100. I. Montvay, H. Satz, Nuovo Cimento A 39, 425 (1977)

101. R. Fiore, R. Page, L. Sertorio, Nuovo Cimento A 44, 531 (1978)

102. R. Fiore, Z. Phys. C 1, 305 (1979)

103. J. Letessier, A. Tounsi, H. Satz, Nuovo Cimento A 45, 580 (1978)

104. H. Satz, in Hadronic Matter at Extreme Energy Density, ed. by N. Cabibbo, L. Sertorio, Erice, 1978 (Plenum Press, New York, 1980), p. 269

105. F. Karsch, D.E. Miller, Phys. Lett. B 79, 259 (1978)

106. T. Celik, H. Satz, Z. Phys. C 1, 163 (1979)

107. N. Cabibbo, L. Sertorio (eds.), in Hadronic Matter at Extreme Energy Density, Erice, 1978 (Plenum Press, New York 1980)

108. W. Nahm, in Hadronic Matter at Extreme Energy Density, ed. by N. Cabibbo, L. Sertorio, Erice, 1978 (Plenum Press, New York 1980), p. 219

109. R. Hagedorn, I. Montvay, J. Rafelski, in Hadronic Matter at Extreme Energy Density, ed. by N. Cabibbo, L. Sertorio. Erice, Italy 1978 (Plenum Press, New York, 1980), p. 49; In this volume we offer a more focused reort, see Thermodynamics of hot nuclear matter 1978 in the statistical Bootstrap model in Melting Hadrons, Boiling Quarks - From Hagedorn Temperature to Ultra-Relativistic Heavy-Ion Collisions at CERN ed. by J. Rafelski (Springer, Heidelberg, 2015)

110. B. Touschek, Nuovo Cimento B 58, 295 (1968)

111. R. Hagedorn, J. Rafelski, Phys. Lett. B 97, 136 (1980)

112. J.I. Kapusta, Nucl. Phys. B 148, 461 (1979)

113. O.K. Kalashnikov, V.V. Klimov, Phys. Lett. B 88, 328 (1979)

114. E.V. Shuriak, Phys. Rep. 61, 71 (1980)

115. K. Kajantie, H. Miettinen, Z. Phys. C 9, 341 (1981)

116. J. Kuti, B. Luckacs, J. Polonyi, K. Szlachanyi, Phys. Lett. B 95, 75 (1980)

117. J.I. Kapusta, Nucl. Phys. B 196, 1 (1982)

118. K. Redlich, Z. Phys. C 21, 69 (1983)

119. R. Hagedorn, J. Rafelski, in Statistical Mechanics of Quarks and Hadrons, Bielefeld Symposium 1980, ed. by H. Satz (North Holland, Amsterdam, 1980), p. 237

120. J. Rafelski, R. Hagedorn, Statistical Mechanics of Quarks and Hadrons, Bielefeld Symposium 1980, ed. by H. Satz (North Holland, Amsterdam, 1980), p. 253

121. V.V. Dixit, F. Karsch, H. Satz, Phys. Lett. B 101, 412 (1981)

122. R. Hagedorn, Z. Phys. C 17, 265 (1983)

123. J. Rafelski, Physics Reports, vol. 88, ed. by M. Jacob, J. Tran Thanh Van (North Holland, Amsterdam, 1982), p. 331

124. J. Rafelski, M. Danos, Perspectives in High Energy Nuclear Collisions, Inst. f. Theor. Phys. Frankfurt Univ. and US Nat. Bureau of Standards NBSIR-83-2725 (1983)

125. M. Jacob, J. Tran Thanh Van (eds.), Physics Reports, vol. 88 (North Holland, Amsterdam, 1982), p. 321

126. W. Shakespeare, Hamlet (1601) 